\title{
Identifying Communication Issues Contributing to the Formation of Chaotic Situation: An AGSD View
}

\author{
Hina Noor ${ }^{1}$, Babur Hayat Malik ${ }^{2}$, Zeenat Amjad ${ }^{3}$, Mahek Hanif $^{4}$ \\ Sehrish Tabussum ${ }^{5}$, Rahat Mansha ${ }^{6}$, Kinza Mubasher ${ }^{7}$ \\ Department of CS and IT, University of Lahore, Gujrat Campus, Pakistan
}

\begin{abstract}
The software can be constructed in many different contexts using various approaches to software creation, Software Development (GSD), Agile Software Development (ASD) and Agile Global Software Development (AGSD) in an ecumenically distributed way (a coalescence of GSD and ASD). This GSD (Global Engenderment of Software) is becoming increasingly important. Although communication is important in the sharing of information between team members, there are additional barriers to multi-site software creation, various time zones and cultures, IT infrastructure, etc., and delays in communication activities that are already problematic. In the case of Agile Global Software Development (AGSD), Agile Global Software Development (AGSD) is much more critical and plays a primary role in interaction and communication. The aim of this paper is to tackle the chaos problems associated with evolution of Agile Global Software (AGSD). We have obtained knowledge from previous works and from web reviews from worldwide, a literature review was conducted. Using a conceptual model, tabulated based on authors, and addressed also, the chaos issues are then illustrated. We identify the most discussed and less discussed issues in the literature. It is consequential to define the chaos issue in order to illustrate the genuine issues that subsisted in AGSD.
\end{abstract}

Keywords-Chaotic situation; chaos; issues; communication; agile; distributed software development; global distributed software development; communication challenges; AGSD

\section{INTRODUCTION}

The aperture among time and location between dispersed software advancement groups is right now considered to integrate to the clamorous Ecumenical Software Development environment (GSD). In addition, as it includes using the agile process, coordination between developers and customers is essential [1]. "Development of software with teams located at various geographical locations, from different national and organizational cultures and time zones" Distributed Software Development (DSD), Ecumenical Software Development is kenned for this kind of development (GSD) [24], [37]. Ecumenical Distributed Software Development or Ecumenical Software Development (GSD) has now become the standard for the Ecumenical market in the software industry. This phenomenon is the product of global economic globalization, which provides the tech industries around the world with a forum for global competitive advantage. In producing quality tech, software companies are competing with each other. With the presence of IT, software creation anywhere in the world, it can be done. Distributed development teams of diverse backgrounds, cultures and languages can be based in different time zones in different geographical areas and still function on the same project [12]. Global team issues shown in Fig. 1.

Over the earlier decade, worldwide programming advancement (GSD), IT rethinking, and reevaluating of business measures have demonstrated yearly development paces of 10-20 percent [5], [6]. The level of offshoring or globalization relies upon the details of the hidden business and what programming is being created. For example, albeit the dispersed IT application made is genuinely circulated, the advancement of straightforward installed programming frameworks actually faces huge difficulties when executing appropriated amplification [13], [14].

The implementation of limber software development has brought paramount amendments to the world of software engineering over the last decay. Many companies and software developers are now utilizing nimble strategies to engender the most efficacious and in the shortest time possible, high-quality product. Extraordinary programming (XP), scrum, lean programming made, include driven amplification (FDD), and DSDM and precious stone approaches are the various kinds of deft procedures utilized by these associations [24]. For the situation of deft ecumenical programming advancement (AGSD), where correspondence assumes an essential part, communication is even more paramount. According to the Supple Manifesto, "Throughout the project, business people and developers must collaborate circadian". In AGSD, communication quandaries were withal widely addressed in the literature [2], [15], which showed that distributed teams, categorically supple teams; rely heavily on communication implements [4]. Albeit several studies have explored the optimal technical stool for fortifying efficient communication in AGSD, it is still an unresolved quandary [3], [16]. As, agile development is elaborated in Fig. 2.

In contrast to geographically dispersed GSD teams, limber development fixates on active face-to-face contact between colocated teams. Limberness provides GSD with both advantages and challenges, such as the difficulty of communication. As interest in using nimble GSD techniques has increased, there has also been an increase in communication literature, as well as communication techniques and limber GSD strategies. There is a desideratum in versatile GSD for researching communication dilemmas and creating or using tools, strategies, and methods to tackle them [7]. By defining, synthesizing and presenting the communication dilemmas of versatile GSD, the purpose of this research paper is to address the above-mentioned gap [17]. 


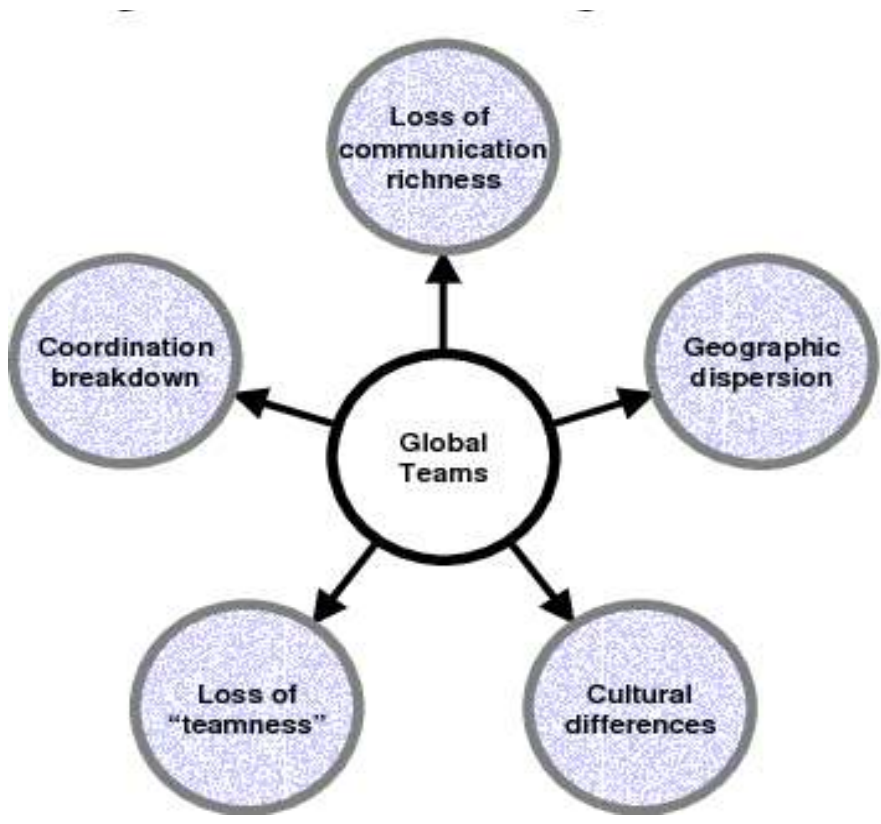

Fig. 1. Global Teams Issues.

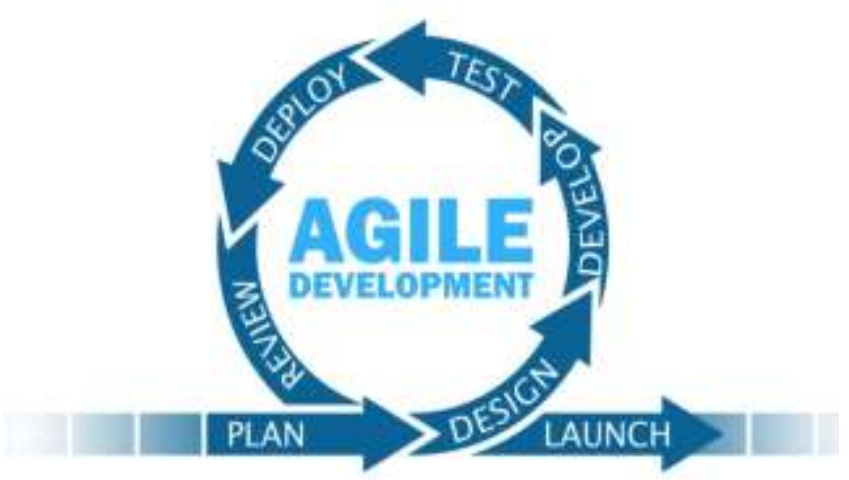

Fig. 2. Agile Development.

This paper is structured as follows. Next, it offers a study of literature. Second, chaos and issues are discussed. Third, it presents and addresses the chaos and issues on communication in AGSD. Fourth, an overview of most discussed, less discussed chaos issues in the literature is presented. Then framework of GSD is elaborated. Determinately, it discusses future work and concludes the whole discussion in the last section

\section{LITERATURE REVIEW}

Agustin Yague and Juan Garbajosa conducted AGSD's Exploratory Communication Research. Instead, in this research work, observations were gathered from three points of view: contact between team members, communication about the status of the production process and the status of the progress of the product under development. The benefits of using media implementations have been explained by team members who assume that teams are co-located in honesty, such as perspicacious boards used by powerful video implements and the accumulation of media implements with centralized repository implements with process-generated data and product features that allow dispersed teams to share information. The results of exploratory research carried out on the effect of communication networks on AGSD are recorded in this paper. Due to distributed generated and traditional meetings suggested by flexible, often short in length and enhanced by face-to-face communication, interaction is critical in AGSD; thus, there are pellucid communication criteria for the amalgamation of the two [1]. The research analyzed the team's views of the facilities utilized for communication support. In this report, communication was addressed in three dimensions: communication between the team, the engineering process and the product under development [1].

Communication problems in the AGSD were identified by Nina Kamarina Kamaruddin, Noor Habibah Arshad and Azlinah Mohamed.

Fig. 3 described the agile methodology step by step. The goal is to resolve the issues associated with communication in Supple Ecumenical Software Development (AGSD). In order to assimilate knowledge from anterior works and from web reviews from practitioners worldwide, a literature survey was conducted. Utilizing a conceptual model, tabulated predicated on authors, and then addressed, the chaos issues are then illustrated. It is paramount to define the chaos issue in order to illustrate the authentic issues that subsisted in AGSD. It will somehow avail researchers and practitioners to identify the genuine quandaries that have arisen in AGSD, predicated on the established chaos quandaries cognate to communication. This paper introduces the chaos challenges encountered by members of the AGSD project team in communication based on existing literature as well as by global practitioners. All the problems that have really arisen in the AGSD setting are seen in the discussion. Our literature survey has established 13 communication-related chaos problems, with the key issues addressed in the literature as well as by the practitioners being various cultures and lack of regular face-to-face interaction [8]. In order to find more communication-related problems in AGSD, longitudinal studies will be carried out in future research.

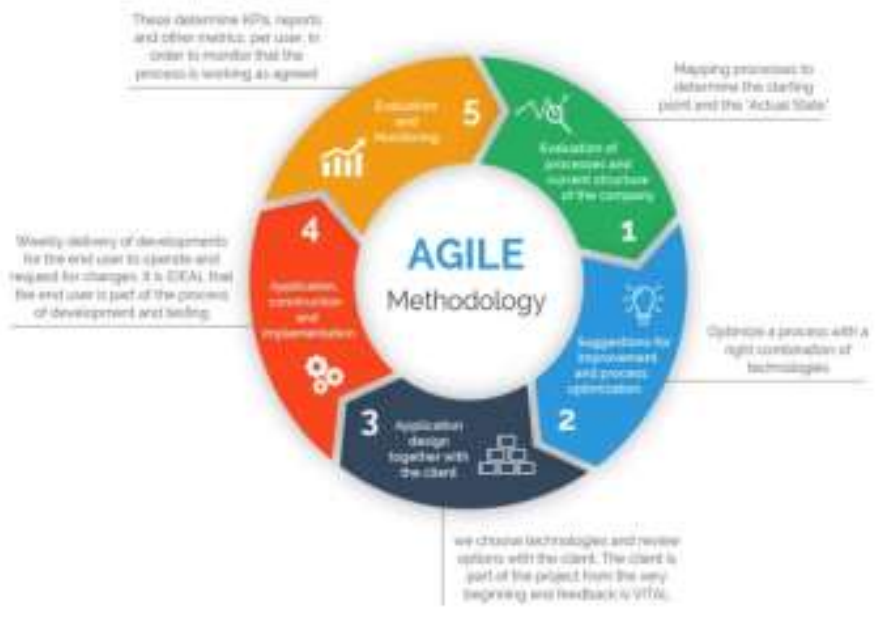

Fig. 3. Agile Methodology. 
Christof Ebert and Marco Kuhrmann in a manner to advance data and innovation move; it summed up points of view from the scholarly community and industry. It depended on an assessment of 10 years of examination and industry participation and experience announced at the IEEE International Conference on Software Engineering (ICGSE) arrangement. The aftereffects of their exploration show that GSE is a profoundly industry-attached field and, subsequently, an enormously goliath extent of ICGSE papers talk about the change of ideas and answers for programming to the ecumenical stage. Collaboration and teams, practices and organization, procurement and supply management, and performance factors were listed as the topics that engendered the most attention from researchers and practitioners. They also looked at emerging developments in GSE to promote more research and industrial cooperation beyond the study of past conferences. They summarized 10 years of ICGSE in their paper, and they searched for the issues explored in the past decade, cumulative information, and patterns. A discussion of the surviving state-of-the-art, focused on recently published studies and discussions by the different ICGSE conference committees, complemented their research. A analysis of the ICGSE papers showed that in the ICGSE conference series, both the conduct of high-quality research and the transition of subsisting software engineering concepts, procedures, practices and implements to the GSE context were addressed. This study has certain disadvantages that need to be discussed. In particular, the study at hand used well-known techniques to reuse validated relegation systems for secondary studies, and implemented systematic data collection and reporting processes. However, because the research focused exclusively on the ICGSE publication pool, they did not claim to show the full image, since they did not include additional publications, such as journal articles or conference papers published at other venues, in their report [6]. The source of their study in literature and only culled open discussions is another drawback. The notion of rigor, significance, and effect additionally affects this.

Yehia Ibrahim Alzoubi and Asif Qumer Gill adopted a SLR approach [4]. In the agile GSD background, and identified communication difficulties. Customized search and cull criteria for literature were first developed and then applied to relegate an accumulation of 449 initially documents. Lastly, for this review, 22 of 449 papers important to this research were chosen. These final 22 papers were analyzed and, in the sense of agile GSD, seven major categories of communication problems were identified. It is expected that the study results of their paper will assist researchers and practitioners to recognize agile GSD's communication challenges and develop methods, techniques and strategies to overcome these challenges. This study identified a range of problems to be tackled in order to build an efficient and effective agile framework GSD. The results of this research have been described in two steps. First, the accentuation of the research and the number of culled papers are registered. Secondly, it reports the information that was analyzed and interpreted from the culled studies to address the study concerns. This research helped us to change the current state of the art of versatile GSD communication issues. This research offers a knowledge base to agile practitioners and academics that have an interest in agile GSD. There are several disadvantages to this analysis, which are homogeneous to all other SLR studies. This paper is limited to the number of studies that have been checked from selected databases. There is a controversy about the use of an inhibited number of culled search databases and a finite number of search strings. This research accumulated papers from renowned databases, and we are completely confident that we have been provided with enough recent literature by the culled databases and search strings to review GSD's new agile communication challenges. Prejudice in the abolition of journals and inaccuracy in the extraction of information are the other paramount constraints of this SLR.

\section{A. Research Questions}

1) What are the chaos issues on Communication in AGSD?

2) What are the most discussed chaos issues on communication in AGSD in the literature?

3) What are the less discussed chaos issues on communication in AGSD in the literature?

\section{CHAOS AND ISSUES}

The study established a root concept (CATWOE) of the structure as visually perceived in order to explicate the "soft" quandary situation resulting from elements that perturb the stable environment of the engendered being studied [9]. Now, the Table I will show the root definition of the factors in the chaotic situation.

To semi-illuminate variables that lead to the creation of a chaotic situation in the creation of an involute system, Chaos theory is briefly demystified while contributors are engendered that lead to some instances of the chaotic situation. The principles of that system are the two key components of chaos theory: 1) Depend on an underlying order, and that 2) No matter how complex they may be. Very simple or small systems and operations can cause very complex behaviors or events. (The situation noted by Edward Lorenz, expressed as a delicate reliance on initial conditions, is this notion.)

TABLE I. ROOT DEFINITION

\begin{tabular}{|l|l|}
\hline $\mathrm{C}=$ Customer & $\begin{array}{l}\text { Software development team, software owner, system } \\
\text { user }\end{array}$ \\
\hline $\mathrm{A}=$ Actors & $\begin{array}{l}\text { Software development teams, project manager, system } \\
\text { owner }\end{array}$ \\
\hline $\mathrm{T}=$ Transformation & $\begin{array}{l}\text { Chaos unwary unstable development environment to } \\
\text { chaos ready a more stable development environment }\end{array}$ \\
\hline $\begin{array}{l}\mathrm{W}=\text { developers } \\
\text { who are aware of }\end{array}$ & $\begin{array}{l}\text { Imminent change will be more prepared to navigate } \\
\text { around a change than they who were caught unwary }\end{array}$ \\
\hline $\mathrm{O}=$ Owner & System owner, government \\
\hline $\mathrm{E}=$ Environment & $\begin{array}{l}\text { Developers with the required know how, technology, } \\
\text { method, and funds }\end{array}$ \\
\hline
\end{tabular}




\section{ChaOs ISSUES ON COMMUNICATION IN AGILE GLOBAL SOFTWARE DEVELOPMENT}

As verbally expressed in the precedent report, it can be considered a challenging task to handle challenges in the context of GSD by cumulating it with agile practices that further perplex things. Due to the distance involved, which somehow causes confusion in the creation process, these problems arise. This was accepted by the fact that the aforementioned uncertainty associated with AGSD problems in software development would have a negative impact on the software outcome. Communication is one of the main quandaries in Agile Global Software Development that has been highlighted in the literature. This difficulty can be considered a concern because it includes distance between locations and various time zones as well as different cultures. [10]. Hence, Fig. 4 will give a clear overview of chaos issues on communication in AGSD.

Nevertheless, communication is regarded as one of the essential elements of GSD, especially in a distributed agile environment where information sharing between team members is possible; understanding customer needs and development activities can be carried out efficiently and effectively [11].

We have listed thirteen issues related to the communication problem in AGSD from the literature. Fig. 6 depicts these problems. In highlighting the real issue that has really occurred in distributed agile programs, these problems are considered relevant.

\section{A. Lack of Frequent Face-to-Face Contact}

The biggest concern illustrated in the literature is the lack of face-to-face interaction. The explanation for this is that the distance from the venue and certain organizations allocated only a small portion of the foreign teams' travel budget. The development teams will try to meet during conferences, corporate training or workshops to connect face-to-face, plus personal meetings during personal meetings during holidays on rare occasions [18]. This problem resulted in the development team relying more on asynchronous communication as well as informal email communication as the right person is not accessible when required [19], [36].

\section{B. Different Project Background}

One of the quandaries illustrated in the literature is project history. Developers from various countries have various types of working culture, a comprehensive example linked to different project contexts, and this may lead to problematic quandaries when cross-border cooperation transpires [20]. Other than that communication turns out to be difficult for various interest groups to understand if agile approaches are new to the development teams involved, it takes time for development members to understand and information is important and should be conveyed to other developers as well as it takes time to change this culture because before that they used plan-driven development [18].

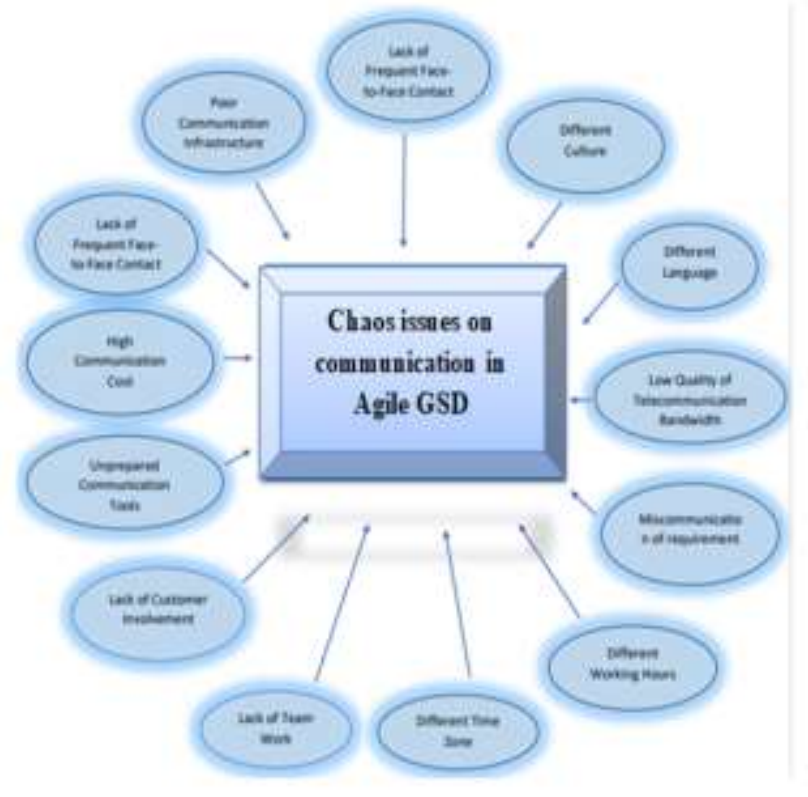

Fig. 4. Chaos Issues on Communication in AGSD.

\section{Different Culture}

One of the most important issues highlighted in literature as well as by practitioners is culture. Some of the cultural-related examples are that some of the members of the offshore team are hesitant to address negative or sensitive topics and only pass on positive data to the onshore team, cultural values and differences of ideology [18], [21], [22]. For example, the understanding of the culture and customs of the offshore teams related to festive seasons or holidays Cultural differences can affect team coordination and communication processes if not carefully handled [7], [8].

\section{Different Language}

Mundanely, distributed construction requires multiple locations or nations. When multiple team members from different countries and multiple members from different countries backgrounds and languages collaborate, it sometimes leads to great frustration. For example, offshore team members who are not native English verbalizers sometimes have arduousness interacting with native English verbalizers in English because of this; meeting often takes longer than mundane because it is arduous for them to communicate their conception [22], [23].

\section{E. Low Quality of Telecommunication Bandwidth}

Telecommunication bandwidth [18] another issue is one that needs to be answered. Often, via a communication medium, because of the context, tone and emotion were disoriented, an excess of time spent to describe things being addressed, and with poor quality of transmission hampering communication implements, communication networks can be slow and unreliable [8]. 


\section{F. Misscommunication of Requirements}

In the creation of software, specifications are considered to be the key component in the production of software that is functional and is continuously changing due to customer changes. If there is a lack of specific customer requirements data, it would have a bad effect on developers where developers have to come up with their own detailed requirements based on their past experience and try to understand what customers need at the same time [8].

\section{G. Different Working Hours}

Differences in time zones are one of the challenges that can be considered major and must be remembered. It improves the contact gap or overhead when there is distance in time zones. This resulted in the difficulties of arranging group meetings outside regular working hours at certain periods of the year due in particular during the winter due to the shorter time as well as the difficulty of holding long meeting instance of Sprint planning meeting [11].

\section{H. Different Time Zones}

One of the major challenges that need to be apperceived is the discrepancies in working hours. It raises the contact distance when there is a disparity in time zones, i.e. communication with the ecumenically dispersed team becomes arduous. In developed countries such as the USA, UK, etc. difficulties in consumer companies are typically expected, the time zone in distributed agile projects is normal [24].

\section{Lack of Team Work}

It is considered a team in distributed growth, even though it includes members of onshore and offshore teams. The software development team needs to collaborate and cooperate thoroughly in order to create a successful and quality product. Often difficulties arise when team members only connect with selected individuals in the team, such as only communicating with the Software Architect, contact is impacted because team members do not want to contribute to interacting with each other. The lack of structured contact can contribute to a decreased team spirit as well. There is also a problem in the growth of team spirit that is located, in two locations, or more particularly when communicating project priorities, goals and domain-specific as well as technical knowledge [8], [24].

\section{J. Lack of Customer Involvement}

Agile approaches are considered to rely more on people's communication than on engaging with clients in particular. The distance of the customers will usually be far away in the Agile distributed sense, resulting in the complexity of regular contact with them [24]. During the creation process, the customer did not offer complete commitment and often the partnership between the two parties is bad because they concentrate more on the process rather than individuals.

\section{K. Unprepared Communication Tools}

Contact is the predominant denotes of interaction For construction teams, both onshore and offshore, and with clients when it requires distance in space and time. It is important to have the right communication tools, but some organizations do not prepare teams with adequate and suitable communication tools, such as video conferencing or web-based conferencing facilities, especially when Scrum meetings are held [11]. It would make it hard to communicate efficiently without these facilities.

\section{High Communication Cost}

The least issues highlighted in the literature are the cost of communication impacting communication gaps [8], [24] where the cost of planning communication facilities is very high and companies need to prepare to spend money on it to provide an efficient means of communication between onshore, offshore and customers located in different locations.

\section{Poor Communication Infrastucture}

In order to encourage the distributed team to communicate with each other, communication infrastructure is considered essential and proper planning must be done. If this problem is not taken care of, there will be a lot of issues later on. For example, moving data to and sharing data with an offshore site typically reveals technical incompatibilities between sites [25]. The distribution of informal news or gossip during informal meetings, coffee breaks or after work meetings may somehow influence countries with poor infrastructure to prohibit rich conversations between team members.

\section{AN OVERVIEW OF MOST DISCUSSED AND LESS DISCUSSED CHAOS ISSUES IN THE LITERATURE}

Table II demonstrates how much the literature discusses these problems.

With the aid of this table, we come to know that the problem is extreme in AGSD communication, i.e. developers and users also face them. We have come to realize that the communication difficulties that are addressed very few times in the literature are what. This gives researchers a new idea that this discussion or research is sufficient or we need to address these problems further because during AGSD they are often most trebly occurring problems. Fig. 5 highlights the mostly discussed communication chaos issues in AGSD.

TABLE II. OVERVIEW OF ISSUES THAT HAVE BEEN IDENTIFIED IN LITERATURE

\begin{tabular}{|l|l|}
\hline ISSUES & LITERATURE \\
\hline $\begin{array}{l}\text { Lack of Frequent Face-To-Face } \\
\text { Contact }\end{array}$ & {$[17],[18],[19],[25],[27],[28],[29]$,} \\
\hline Different Project Background & {$[8],[19],[26],[27]$} \\
\hline Different Culture & {$[24],[25],[27],[28],[29]$,} \\
\hline Different Language & {$[8],[17],[24],[31]$} \\
\hline $\begin{array}{l}\text { Low quality of telecommunication } \\
\text { background }\end{array}$ & $[18], 28],[30]$ \\
\hline $\begin{array}{l}\text { Miscommunication of } \\
\text { Requirements }\end{array}$ & {$[19],[32]$} \\
\hline Different working hours & {$[8],[17],[27,[28],[29]$} \\
\hline Different time zones & {$[11],[24],[30],[31],[34]$} \\
\hline Lack of team work & {$[11],[25]$} \\
\hline Lack of customer involvement & {$[29],[30],[33]$} \\
\hline Unprepared communication tools & {$[11],[28],[30],[31]$} \\
\hline High communication cost & {$[25],[28]$} \\
\hline Poor communication infrastructure & {$[25],[28]$} \\
\hline
\end{tabular}




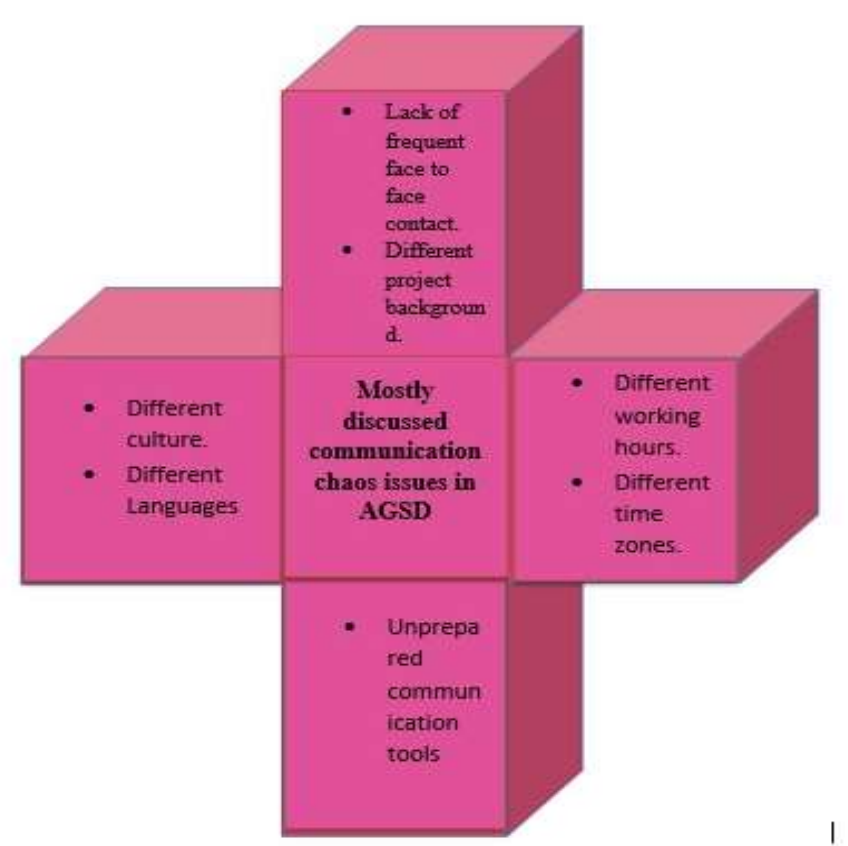

Fig. 5. Mostly Discussed Communication Chaos Issues in AGSD.

Based on the above statistics, there are two key problems that the researcher has regularly addressed and encountered by the practitioners. Different cultures and the lack of customary face-to-face interaction between scattered development teams are linked to the quandaries. Various operating hours or various time zones are also a problem often stated in the literature and by practitioners because of different geographical locations. This is accompanied by lack of confidence or ability to interact between team members, different histories of projects and different languages used between different countries. Fig. 6 highlights the less discussed chaos issues in AGSD.

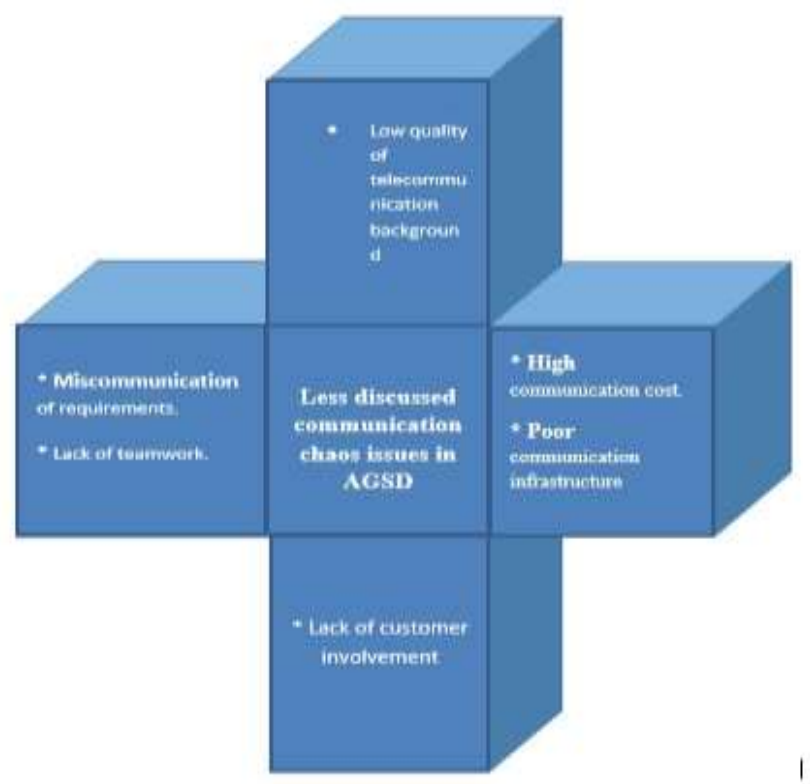

Fig. 6. Less Discussed Communication Chaos Issues in AGSD.
Lack of customer participation, efficiency of telecommunication capacity, communication costs, communication resources, lack of engagement or coordination by development teams, miscommunication of specifications and, last but not least, communication infrastructure or technological incompatibilities are the least listed problems that have been addressed.

\section{Framework of Global SOFTWARE DEVELOPMET}

Julia M. Kotlarsky and Jos van Hillegersberg [35] have researched and benchmarked (as a theoretical background) ecumenically distributed projects, and have developed an Ecumenical Software Development Project Structure that defines functional areas in which distributed teams cooperate during different project phases. During empirical data collection, they used these functional areas as a framework for group communication problems, teamwork and cooperation, and communication patterns and implements that we define. The framework describes and links different elements of projects in ecumenical software: product, methodology, project organization (i.e. individuals) and plans, and efficient planning, communication and control activities.

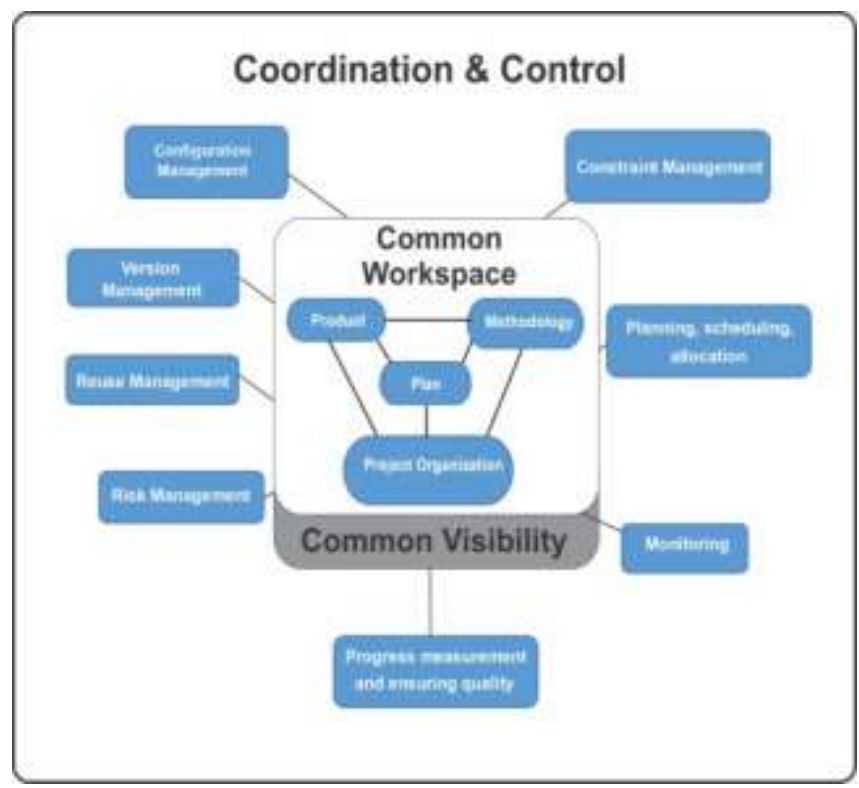

Fig. 7. Framework for Negotiation and Reaching of Consensus.

This framework shown in Fig. 7 will definitely help in the handling of chaotic situation in the agile GSD. So we can say that GSD teams should follow this framework in communication, coordination and control.

\section{CONCLUSION AND FUTURE WORK}

Over the last decade, global distribution for software production has become popular. There are a number of economic and technological trends that are expected to push distributed software development growth further. On the technical side, recent progress in information and communication systems makes it possible to work on software development projects in distributed modes through abstract distance perception. 
In summary, this paper presents the chaos of communication based on the latest literature encountered by AGSD project team members. All the problems that have really arisen in the AGSD setting are seen in the discussion. Our literature survey found 13 communication-related chaos problems, with various cultures and lack of regular face-to-face interaction being the key issues addressed in the literature. The results of this review shows that there is need to be discuss these chaos issues more that are ignored by the researchers in the literature as these chaos issues are equally responsible in the formation of chaotic situation. At the end we also present a framework of coordination and control that will help to mitigate communication chaos issues in Agile GSD.

There is a desideratum in agile GSD to research communication issues and create or use implements, methods, and tactics to tackle them. Future studies would recommend the implementation in a distributed world of versatile approaches by designating timely implementing resources to alleviate these problems of chaos.

The results show that the key risk problem is communication in Agile Global Software development. The contributions of this paper may enable the scholars to propose and validate an enhanced approach to solve the problems of risk communication issues in the Agile GSD environment and assist practitioners in choosing the most effective and applicable method based on the Agile GSD project requirement. In the future, we will also concentrate on contributing to the field of understanding of software engineering management and software engineering models and methods.

\section{REFERENCES}

[1] Yagüe, Agustin, et al. "An exploratory study in communication in Agile Global Software Development." Computer Standards \& Interfaces 48 (2016): 184-197.

[2] Jalali, Samireh, and Claes Wohlin. "Global software engineering and agile practices: a systematic review." Journal of software: Evolution and Process 24.6 (2012): 643-659.

[3] da Silva, Fabio QB, et al. "Challenges and solutions in distributed software development project management: A systematic literature review." 2010 5th IEEE International Conference on Global Software Engineering. IEEE, 2010.

[4] Alzoubi, Yehia Ibrahim, and Asif Qumer Gill. "Agile global software development communication challenges: A systematic review." Proceedings-Pacific Asia Conference on Information Systems, PACIS 2014. 2014.

[5] TaKeaways, Key. "The Forrester Wave: B2C Global Commerce Service Providers, Q1 2015." (2015).

[6] Ebert, Christof. Global software and IT: a guide to distributed development, projects, and outsourcing. John Wiley \& Sons, 2011.

[7] Gill, Asif Qumer, Deborah Bunker, and Philip Seltsikas. "Evaluating a communication technology assessment tool (Ctat): a case of a cloud based communication tool." Proceedings-Pacific Asia Conference on Information Systems, PACIS 2012. 2012.

[8] Kamaruddin, Nina Kamarina, Noor Habibah Arshad, and Azlinah Mohamed. "Chaos issues on communication in agile global software development." 2012 IEEE Business, Engineering \& Industrial Applications Colloquium (BEIAC). IEEE, 2012.

[9] Othman, Marini, Abdullah Mohd Zin, and Abdul Razak Hamdan. "Constructing a chaos proofing pre-development framework to manage chaos in a chaos-prone systems development environment." 2008 8th IEEE International Conference on Computer and Information Technology. IEEE, 2008.
[10] Jalali, Samireh, and Claes Wohlin. "Agile practices in global software engineering-A systematic map." 2010 5th IEEE International Conference on Global Software Engineering. IEEE, 2010.

[11] Dorairaj, Siva, James Noble, and Petra Malik. "Effective communication in distributed Agile software development teams." International Conference on Agile Software Development. Springer, Berlin, Heidelberg, 2011.

[12] Stray, Viktoria, and Nils Brede Moe. "Understanding coordination in global software engineering: A mixed-methods study on the use of meetings and Slack." Journal of Systems and Software 170 (2020): 110717.

[13] Khan, Arif Ali, and Muhammad Azeem Akbar. "Systematic literature review and empirical investigation of motivators for requirements change management process in global software development." Journal of Software: Evolution and Process 32.4 (2020): e2242.

[14] Barbosa, Hualter O., et al. "Developing a release management tool to support global software development: an experience report on Android platform." Proceedings of the 15th International Conference on Global Software Engineering. 2020.

[15] Camara, Rafael, et al. "Agile Global Software Development: A Systematic Literature Review." Proceedings of the 34th Brazilian Symposium on Software Engineering. 2020.

[16] Vallon, Raoul, et al. "Systematic literature review on agile practices in global software development." Information and Software Technology 96 (2018): 161-180.

[17] Podari, Zuriyaninatasa, et al. "Systematic Literature Review on Global Software Development Risks in Agile Methodology." 2020 8th International Conference on Information Technology and Multimedia (ICIMU). IEEE, 2020.

[18] Lee, Seiyoung, and Hwan-Seung Yong. "Distributed agile: project management in a global environment." Empirical Software Engineering 15.2 (2010): 204-217.

[19] Lehtonen, Ismo. "Communication challenges in agile global software development." University of Helsinki, Department of Computer Science, Faculty of Science (2009).

[20] Damian, Daniela, et al. "Awareness in the wild: Why communication breakdowns occur." International Conference on Global Software Engineering (ICGSE 2007). IEEE, 2007.

[21] ul Haq, Sami, et al. "Issues in global software development: A critical review." Journal of Software Engineering and Applications 4.10 (2011): 590.

[22] Marinho, Marcelo, Alexandre Luna, and Sarah Beecham. "Global software development: practices for cultural differences." International Conference on Product-Focused Software Process Improvement. Springer, Cham, 2018.

[23] Dorairaj, Siva, James Noble, and Petra Malik. "Understanding team dynamics in distributed Agile software development." International conference on agile software development. Springer, Berlin, Heidelberg, 2012.

[24] Kaur, Pawanpreet, and Sumit Sharma. "Agile software development in global software engineering." International Journal of Computer Applications 97.4 (2014).

[25] Kornstadt, Andreas, and Joachim Sauer. "Tackling offshore communication challenges with agile architecture-centric development." 2007 Working IEEE/IFIP Conference on Software Architecture (WICSA'07). IEEE, 2007.

[26] B. Lofland, iProject risk or issue?,h PM Technix. 12-2010.

[27] Bose, Indranil. "Lessons learned from distributed agile software projects: A case-based analysis." Communications of the Association for Information Systems 23.1 (2008): 34.

[28] Sauer, Joachim. "Agile practices in offshore outsourcing-an analysis of published experiences." Proceedings of the 29th information systems research seminar in Scandinavia, IRIS. Vol. 29. 2006.

[29] Dullemond, Kevin, Ben van Gameren, and Rini van Solingen. "How technological support can enable advantages of agile software development in a GSE setting." 2009 Fourth IEEE International Conference on Global Software Engineering. IEEE, 2009. 
[30] Hossain, Emam, Muhammad Ali Babar, and Hye-young Paik. "Using scrum in global software development: a systematic literature review." 2009 Fourth IEEE International Conference on Global Software Engineering. Ieee, 2009.

[31] I would like to know what are the issues/challenges/problems that exist in Agile Global Software Development. Care to share your experiences?,h Agile Alliance. Dec-2010.

[32] Korkala, Mikko, Minna Pikkarainen, and Kieran Conboy. "Distributed agile development: A case study of customer communication challenges." International Conference on Agile Processes and Extreme Programming in Software Engineering. Springer, Berlin, Heidelberg, 2009.

[33] Korkala, Mikko, and Pekka Abrahamsson. "Communication in distributed agile development: A case study." 33rd EUROMICRO Conference on Software Engineering and Advanced Applications (EUROMICRO 2007). IEEE, 2007.
[34] Layman, Lucas, et al. "Essential communication practices for Extreme Programming in a global software development team." Information and software technology 48.9 (2006): 781-794.

[35] Kotlarsky, Julia M., Kuldeep Kumar, and Jv Hillegersberg. "Coordination and collaboration for globally distributed teams: the case of componentbased/object-oriented software development." Proceedings of International Workshop on Global Software Development (ICSE 2002). 2002.

[36] Shameem, Mohammad, et al. "Taxonomical classification of barriers for scaling agile methods in global software development environment using fuzzy analytic hierarchy process." Applied Soft Computing 90 (2020): 106122.

[37] Sinha, Richa, Mohammad Shameem, and Chiranjeev Kumar. "SWOT: strength, weaknesses, opportunities, and threats for scaling agile methods in global software development." Proceedings of the 13th innovations in software engineering conference on formerly known as India software engineering conference. 2020. 